\title{
Mitigando el cambio climático desde la educación infantil: una investigación-acción
}

\author{
Mitigating Climate Change from Early Childhood Education: an action-research \\ Mitigando as Mudanças Climáticas desde a Educação Infantil: uma pesquisa-ação
}

\author{
Sandra Pérez Lisboa \\ sandra.perez@upla.cl \\ Universidad de Playa Ancha - Chile ${ }^{1}$ \\ https://orcid.org/0000-0002-0389-7242
}

\begin{abstract}
RESUMEN
El cambio climático afecta a todos los países produciendo un impacto negativo en su economía, las comunidades, el entorno natural y las personas. La educación desde la infancia cumple un rol fundamental, para que los niños y niñas se involucren en diversas acciones habituales que les permita actuar consiente y reflexivamente en una sociedad de crecientes cambios científicos y tecnológicos. Esta investigación-acción tuvo como objetivo desarrollar conocimientos, actitudes y valores, para comprender los efectos del cambio climático en los niños y niñas del nivel Medio Mayor de un Jardín Infantil de la comuna de San Felipe, Chile. Por medio de una investigación- acción participativa, la estudiante de práctica profesional realizó diversas experiencias de aprendizajes a 28 niños y niñas, los cuales aprendieran medidas de mitigación y de adaptación al cambio climático en el Valle de Aconcagua. Los resultados de las experiencias planificadas permitieron desarrollar actitudes y valores en los infantes y comunidad educativa. Asimismo, abrir espacios de discusión en torno a la responsabilidad de los centros educativos de educación infantil, sobre el cambio climático y cómo éste afecta la paz de las comunidades.
\end{abstract}

Palabras-Claves: Cambio climático, educación infantil, adaptación y mitigación.

\begin{abstract}
Climate change affects all countries, producing a negative impact on their economy, communities, natural environment and people. Education from childhood plays a fundamental role, so that children are involved in various habitual actions that allow them to act consciously and reflectively in a society of increasing scientific and technological changes. The objective of this action research was to develop knowledge, attitudes and values, to understand the effects of climate change on boys and girls of the Upper Middle level of a Kindergarten in the commune of San Felipe, Chile. Through participatory action research, the professional practice student carried out various learning experiences with 28 boys and girls, who learned mitigation and adaptation measures to climate change in the Aconcagua Valley. The results of the planned experiences allowed the development of attitudes and values in infants and the educational community. Likewise, open spaces for discussion around the responsibility of early childhood education centers, on climate change and how it affects the peace of communities.
\end{abstract}

Keywords: Climate change, early childhood education, adaptation and mitigation

\section{RESUMO}

A mudança climática afeta todos os países, produzindo um impacto negativo em sua economia, comunidades, meio ambiente natural e pessoas. A educação desde a infância desempenha um papel fundamental, para que as crianças se envolvam em diversas ações habituais que lhes permitem atuar de forma consciente e reflexiva em uma sociedade em crescentes mudanças científicas e tecnológicas. O objetivo desta pesquisa-ação foi desenvolver conhecimentos, atitudes e valores para compreender os efeitos da mudança climática em meninos e meninas do Nível Médio Alto de um Jardim de Infância na comuna de San Felipe, Chile. Por meio da pesquisa-ação participativa, o aluno de prática profissional realizou diversas experiências de aprendizagem com 28 meninos e meninas, que aprenderam medidas de mitigação e adaptação às mudanças climáticas no Vale do Aconcágua. Os resultados das experiências planejadas permitiram o desenvolvimento de atitudes e valores nos bebês e na comunidade educacional. Da mesma forma, abrir espaços de discussão em torno da responsabilidade dos centros de educação infantil, sobre as mudanças climáticas e como elas afetam a paz das comunidades.

\footnotetext{
${ }^{1}$ Este proyecto fue financiado por la Unidad de Desarrollo Docente de la Universidad de Playa Ancha - Chile.
} 
Palavras-chave: Mudanças climáticas, educação infantil, adaptação e mitigação.

\section{INTRODUCCIÓN}

El desarrollo sostenible se define como "la satisfacción de las necesidades de la generación presente sin comprometer la capacidad de las generaciones futuras para satisfacer sus propias necesidades" (Organización de las Naciones Unidas [ONU], 1987, p. 59). El desarrollo sostenible surge "para proteger el planeta contra la degradación, incluso mediante el consumo y la producción sostenibles, la gestión sostenible de sus recursos naturales y medidas urgentes para hacer frente al cambio climático" (ONU, 2015, p.2). Para ello la ONU en el año 2015, lanza la Agenda 2030 para el Desarrollo Sostenible (ODS), la que establece 17 Objetivos de Desarrollo Sostenible (ODS) y 169 metas que permitirán ir avanzando hacia la sostenibilidad económica, social y ambiental. Por medio de esta agenda se da un hito importante, ya que se manifiesta la necesidad de erradicar la pobreza con el desarrollo sostenible.

En Chile, el desarrollo sostenible se ha estado trabajando en distintos ámbitos, a nivel educativo se ha incorporado en el currículo de todos los niveles. Para Macedo (2006), por medio de la educación se contribuye a la formación de valores, conocimientos y comportamientos en los ciudadanos, lo que permitirá cimentar un mundo sostenible. Por eso es fundamental que en la formación inicial docente se mejoren los procesos de enseñanza y aprendizajes de las ciencias a nivel escolar (Asencio, 2013). Según Schindel (2012), los profesores y los estudiantes al crear proyectos de acción ambiental importantes para la comunidad, demuestran el empoderamientos de los estudiantes, para trabajar la educación de la ciencia de la justicia social. Por consiguiente, la educación científica favorece la formación y la comprensión de los estudiantes de los problemas ambientales, para avanzar en una sociedad consiente del impacto que provoca el cambio climático en todos los seres vivientes.

En este contexto, la actualización de las Bases Curriculares de Educación Parvularia (en adelante BCEP), desarrolla de manera transversal en los diferentes ámbitos de aprendizaje, la educación ambiental. Según las BCEP “...el enfoque de la sostenibilidad es clave, en tanto conlleva favorecer aprendizajes que permitan a niñas y niños comprender las potencialidades, oportunidades y riesgos que implica sostener la vida humana en un planeta con recursos limitados" (Subsecretaria de Educación Parvularia, 2017, p. 80).

Ahora bien, el cambio climático como consecuencia del "macro problema real" (Estenssoro, 2010, p. 59), provocado por la concentración de CO2, amenaza a Chile y una de las provincias más afectadas en el valle del Aconcagua. En este Valle el cambio climático se puede observar con las sequías que han habido desde hace una década y los cambios en el caudal del río Aconcagua, que se encuentra cerca de sus mínimos históricos. La escasez hídrica ha traído consecuencias negativas a la población del sector agrícola y ganadero, así también a la población en general que vive en el valle, ya que gran parte de ellos y ellas aun trabajan en el sector agrícola. Para enfrentar estas dificultades según la ONU, es necesario "la elaboración y aplicación de programas de educación y sensibilización del público sobre el cambio climático y sus efectos" (1992, p. 25). Como también"...fomentar el tipo adecuado de competencias, actitudes y comportamientos que llevarán al crecimiento sostenible e inclusivo” (UNESCO, 2017, p.1).

En consecuencia con lo anterior, por los efectos del cambio climático en el Valle Aconcagua y la urgencia de programas educativos que señala la ONU y la UNESCO, se propone este proyecto de intervención pedagógica. El proyecto de intervención pedagógica se realizó en el Jardín Infantil 'El Peneca' perteneciente a la Junta Nacional de Jardines Infantiles (en adelante JUNJI). El objetivo de esta investigación fue desarrollar conocimientos, actitudes y valores, para comprender los efectos 
del cambio climático en los niños y niñas del nivel Medio Mayor de un Jardín Infantil de la comuna de San Felipe, Chile. Este establecimiento educacional está incorporando prácticas de sostenibilidad, además se está acreditando como Jardín Infantil medio ambiental por el Departamento de medio Ambiente del Municipio de San Felipe.

\section{FUNDAMENTACIÓN TEÓRICA}

Los sectores económicos de exportación de Chile son la minería, forestación y la agricultura, la sobreexplotación de estos recursos naturales, ha provocado un agotamiento en: degradación de suelo; escasez hídrica; árboles frutales como cítricos, paltos y vides. Esta sobreexplotación ha tenido además un costo social, afectando la calidad de vida de las personas que viven en estas localidades, causando enfermedades por los daños en el medio ambiente. Según Préndez y Calderón (2012), los impactos en el medio ambiente de estas localidades, no han sido tratados como problemas o conflictos de carácter público.

Por otro lado Sabatini (1994), señala que si bien hay costos ambientales y sociales para las personas que viven en las zonas cercanas a empresas agrícolas o mineras, se generan discusiones entre los actores de una localidad. En estas discusiones se enfrentan tres actores principales: las empresas que ocupan los recursos ambientales; la comunidad o grupo de esta, en defensa del medio ambiente; y una agencia pública mediadora en los conflictos ambientales. Al respecto Chile, ha favorecido en las comunidades la capacidad de constituirse como actores autónomos frente al estado y partidos políticos, para que lleve a la discusión política los problemas ambientales locales y que estos sean tratados como problemas políticos-públicos.

El año 2010 se crea el Ministerios del Medio Ambiente (en adelante MMA), a través de la Ley 20417. Este Ministerio colabora con el jefe de Estado en el diseño y aplicación de políticas, programas y planes sobre el medio ambiente, su conservación y protección y promoviendo el desarrollo sustentable. Con respecto al cambio climático, este Ministerio tiene la División de Cambio Climático, cuya misión es:

Contribuir en el desarrollo sustentable y resiliente a los impactos del cambio climático y en una economía baja en carbono del país, a través de la integración e impulso de más y mejores políticas públicas sectoriales que permitan a nivel local enfrentar el cambio climático e implementar acciones de mitigación; que sirvan a su vez, de ejemplo a nivel global (MMA, en línea).

Por otra parte esta División debe orientar y efectuar la Convención de Viena y el Protocolo de Montreal, de las sustancias destructoras de la capa de ozono, favoreciendo acciones de protección de la capa de ozono para enfrentar el cambio climático. Según el MMA (2018), la mayor parte del territorio está afectado por la contaminación del medio ambiente, la combustión de leña sigue siendo la principal emisora de MP2, 5 para el año 2015, mientras que las fuentes móviles, termoeléctricas y otros procesos industriales son los mayores responsables de emisiones de óxidos de nitrógeno (NOX). Las emisiones de dióxido de azufre (SO2) están lideradas por las fundiciones y termoeléctricas.

Otro problema que afecta al territorio es la escasez hídrica, entre 2008 y 2017, la Dirección General de Aguas, declaró 82 zonas entre las regiones de Atacama y Aysén, concentradas principalmente en las regiones de Coquimbo, Valparaíso, Maule y Metropolitana. Por ello que el Ministerio de Agricultura el año 2008, declaró emergencia agrícola a 225 comunas del país, por la sequía que se ha reportado en una extensa geografía, lo que se ha mantenido configurando una "Megasequía".

En los últimos años la mitigación en Chile, ha estado marcada principalmente por la cooperación intersectorial y el compromiso que ha adoptado el estado de Chile, en la construcción 
de políticas públicas que promuevan una economía baja en emisiones de carbono. Prueba de esto, es el lanzamiento del Plan de Acción Nacional de Cambio Climático 2017-2022 y el Plan de Mitigación del Sector Energía, entre otras.

A nivel educativo el MMA, tiene el Sistema Nacional de Certificación de Establecimientos Educativos (en adelante SNCAE). Este sistema es un programa coordinado por la Unesco, el Ministerio de Educación, la División de Educación Ambiental del Ministerio del Medio Ambiente, la Asociación Chilena de Municipalidades (ACHM), la Corporación Nacional Forestal (CONAF) y la Dirección General de Aguas (DGA). El programa fomenta la educación ambiental a través de acciones complementarias para la gestión ambiental local. El SNCAE aprueba y fomenta los proyectos educativos, difusión y promoción ambiental para que todas y todos vayan desarrollando la conciencia de sustentabilidad. El sistema otorga una certificación a los establecimientos educacionales del país que implementen metodologías y/o estrategias adecuadas a su entorno socioambiental, acreditación que se da en tres niveles: Básico, Medio y Excelencia. Actualmente son 110, los jardines infantiles de la JUNJI de administración directa y Vía Transferencia de Fondos (VTF), los que tienen esta certificación.

\subsection{Educación Parvularia}

Respecto a la Educación Parvularia, la actualización de las BCEP, señala que “...frente a problemáticas asociadas a fenómenos socio ecológicos que afectan la sostenibilidad ambiental, resulta fundamental incorporar desde los primeros años acciones educativas cotidianas para abordar estos desafíos que enfrenta la sociedad" (Subsecretaria de Educación Parvularia, 2017, p.11). Este referente curricular considera que las experiencias educativas para los niños y niñas deben ser integradoras e inclusivas, con ambientes potenciadores y que permitan la participación activa para que favorezcan múltiples aprendizajes. Asimismo, este accionar pedagógico implica el desarrollo integral, el respeto a la persona en sus diversas dimensiones, el cuidado del medio natural y cultural, la paz, la justicia, revelando el bien común con el fin de atestar la calidad de vida para las generaciones presentes y futuras.

Por otro lado, el Ministerio de Educción señala que la Educación para el Desarrollo Sustentable debe considerar la equidad de género, la convivencia, el conocimiento y actitudes positivas a la naturaleza, entre otros aspectos. Para lo cual la participación de toda la comunidad educativa es fundamental, esto permitirá “...generar impactos positivos en lo social, cultural, económico y ambiental desde el nivel local” (2013, p. 6), así el establecimiento educativo podrá ser un agente de cambio en la comunidad que este inserto.

Consecuentemente con lo anterior, la Juna Nacional de Jardines Infantiles considera que los primeros aprendizajes de los niños y niñas son fundamentales, ya que determinan la calidad de vida presente y futura de estos y estas (JUNJI, en línea). Por ello se les ofrece diversas instancias para que enriquezcan la forma de ver el mundo, para que puedan comunicar y expandir su natural curiosidad de descubrir, explorar, indagar, valorar y cuidar el entorno cercano. El objetivo que esta institución se plantea es:

Que educadoras, técnicas, agentes educativos y familias puedan favorecer y fortalecer el vínculo de niños y niñas con el medio natural, social y cultural, conectándolos con la valoración de la naturaleza, la humanidad, el aprecio por el planeta y sus recursos como un bien necesario de proteger y preservar, para el buen vivir de las generaciones presentes y futuras (Toro y Ugarte, 2018, p. 9).

Para lo cual, el currículo es un enfoque holístico e integral: educadoras, técnicas y agentes educativos sitúan a los niños y niñas en el centro, observando su entorno y vínculos como actores de esta sociedad, del territorio y la comunidad que habitan. "La familia, el medio social, las 
necesidades de aprendizaje, el género, la cultura, el pensamiento lógico matemático, los requerimientos de una vida saludable, la comunicación, el afecto, el juego, la creatividad, coexisten en el párvulo armónicamente" (JUNJI, 2015, p. 8).

En relación a la sostenibilidad la JUNJI, establece compromisos con el MMA, elaborando un Plan de Trabajo para la Protección del Medioambiente cuyo objetivo general es "promover en las comunidades educativas de la JUNJI el respeto al medioambiente y el uso racional de los recursos naturales, como expresión concreta de la solidaridad con las futuras generaciones" (ídem).

\subsection{Jardín Infantil El Peneca}

El jardín infantil El Peneca, fue fundado el 3 de agosto de 1976, para responder a la necesidad de las mujeres que se integraron de forma masiva al campo laboral. Sus inicios se gestaron por la Asociación de Agricultores de San Felipe y las autoridades de la época. Funcionó en un local que fue expropiado al partido Radical en el centro de la ciudad, una vez que el partido Radical recuperó el inmueble la JUNJI debió pagar arriendo hasta el año 2014. En marzo del año 2015, la institución logró ubicar un nuevo local en conjunto con la municipalidad y la comunidad, ubicándolo en un sector urbano de alta densidad poblacional, como lo es la Villa El Descanso y cercana a la Villa El Señorial y el sector de La Troya.

Este establecimiento atiende los niveles educativos de Sala Cunas y Niveles Medios, con una capacidad de 126 niños y niñas de 3 meses a 4 años de edad, que pertenecen en su mayoría a familias monoparentales y extensas. La misión que tiene la JUNJI para los Jardines Infantiles es:... entregar Educación Parvularia de calidad y bienestar integral a niños y niñas preferentemente entre 0 y 4 años de edad, priorizando las familias con mayor vulnerabilidad socioeconómica, a través de una oferta programática diversa y pertinente a los contextos territoriales” (JUNJI, en línea).

Para ello, en cada establecimiento la mediación pedagógica se acompaña de ambientes que propicien el descubrimiento, el asombro y la investigación por parte de niños y niñas. Estos ambientes potenciadores, facilitan la autonomía de los infantes y permite desarrollar la creatividad de educadoras y técnicos.

\section{PROCEDIMENTOS METODOLÓGICOS}

Esta investigación de carácter cualitativa tuvo como objetivo desarrollar conocimientos, actitudes y valores, para comprender los efectos del cambio climático en los niños y niñas del nivel Medio Mayor, de un Jardín Infantil de la comuna de San Felipe, Chile. Las investigaciones cualitativas tienen como principal elemento la acción indagatoria y la sensibilización con el ambiente en el que esta se realiza (Hernández, Fernández \& Baptista, 2014), estos dos elementos se han encontrado presentes durante todo el proceso de esta investigación.

\subsection{Tipo de investigación}

El tipo de investigación cualitativa utilizado para este estudio, es el de investigación-acción que según Sandín permite "propiciar el cambio social, transformar la realidad y que las personas tomen conciencia de su papel en ese proceso de transformación" (2003, p.161). Proceso de transformación que se pretendió desarrollar al educar a niños y niñas, educadoras, técnicos, familia y comunidad en el cambio climático. El tipo de estudio que se utilizó fue la investigación-acción participativo, según Creswell "implica una inclusión completa y abierta de los participantes en el 
estudio, como colaboradores" (2012, p. 583), en este caso, fue la estudiante de práctica profesional la que se incluyó al establecimiento, implementando el proyecto de intervención pedagógica.

El diseño de la investigación-acción según Stringer (1999) tiene tres fases. La primera fase es observar el problema y recopilar datos acerca de este. La segunda fase es pensar y analizar críticamente los datos recopilados e interpretarlos. La tercera fase es actuar, realizando acciones que busquen resolver el problema, implementando mejoras. Todas estas fases, se dan de manera cíclica, una y otra vez, hasta que la mejora se introduce satisfactoriamente. A continuación se detallan cada una de las fases realizada en esta investigación- acción:

3.1.1 Fase de observar: al incorporarse la estudiante a su práctica profesional, la educadora guía le informa que el Jardín Infantil está realizando las acciones para obtener la certificación ambiental del SNCAE. Por ello le propone a la estudiante realizar un proyecto de educación ambiental. La estudiante comienza a observar las problemáticas del Valle Aconcagua.

3.1.2 Fase de pensar: Luego de la indagación y recopilación de datos, la practicante en conjunto con la educadora guía, directora del establecimiento y supervisora de práctica deciden realizar el proyecto 'Conociendo el cambio climático en el Jardín Infantil El Peneca. Este proyecto de intervención pedagógica, tuvo como objetivo desarrollar conocimientos, actitudes y valores, para comprender los efectos del cambio climático en los niños y niñas, familia y comunidad circundante del establecimiento. El nivel Medio Mayor tiene una matrícula de 28 niños y niñas, entre 2 a 2años y 11 meses de edad.

3.1.3 Fase de actuar: Para dar cumplimiento al objetivo planteado en la fase de pensar, el proyecto de intervención pedagógica trabajó con las siguientes tres líneas de acción:

3.1.3.1 Niños y niñas: Se implementaron 34 experiencias pedagógicas (EP), en ellas se trabajaron los temas de i) concepto de cambio climático (4 EP); ii) efectos del cambio climático en el Valle de Aconcagua ( $8 \mathrm{EP})$; iii) escasez hídrica (9 EP); iv) mitigaciones que se realizan en el Valle de Aconcagua (13 EP).

Estas experiencias pedagógicas fueron apoyadas con la pizarra digital, para Pérez-Lisboa "con el uso de esta herramienta se obtienen innumerables posibilidades de innovar en las prácticas pedagógicas, con el propósito de motivar al estudiantado en el proceso de aprender, teniendo en cuenta la diversidad, características y necesidades" (2017, p. 5).

3.1.3.2 Familia: El trabajo con la familia se realizó por medio de dos reuniones de padres y apoderados en las que participó la estudiante de práctica profesional y el envío de trípticos informativos. En estas reuniones se educó en el manejo de residuos en casa y el uso eficiente del agua. En los tres trípticos informativos se trataron los siguientes temas: en el primer tríptico se educaba sobre el manejo de residuos sólidos en sus hogares; en el segundo tríptico se daba a conocer la importancia del agua para su valoración y comprensión; y en el tercer tríptico se informó sobre las acciones que pueden realizar en sus hogares para el ahorro de energía.

3.1.3.3 Comunidad: Para educar a la comunidad circundante del establecimiento educativo, los niños y niñas prepararon folletos informativos, los cuales fueron entregados por ellos en las casas y acompañados por las educadoras del nivel y la estudiante en práctica.

\subsection{Instrumento para recoger los datos}

Los instrumentos de recogida de datos utilizados en la investigación han sido los siguientes: 
1. La Observación participante: Esta técnica permite al investigador tener "experiencias directas con los participantes y el ambiente" (Hernández, Fernández y Baptista, 2014, p. 417). Con estas experiencias la estudiante en práctica, observó como los niños y niñas, la familia y el entorno fueron comprendiendo los efectos del cambio climático.

2. Encuesta: Este instrumento que se hace de forma escrita y por medio de papel es "contentivo con una serie de preguntas. Se denomina auto administrado porque debe ser llenado por el encuestado sin intervención del encuestador" (Arias, 2016, p.74). La encuesta fue enviada a la familia para que ellas dieran a conocer los conceptos que los niños y niñas iban desarrollando sobre los efectos del cambio climático en sus vidas.

\subsection{Proceso de aplicación de los instrumentos}

Para realizar la aplicación de los instrumentos, se realizó una reunión de padres y apoderados donde se explicó el propósito del estudio y la forma en que se iban a recoger los datos. Los padres, madres y apoderados firmaron el consentimiento informado, antes de aplicar el proyecto.

\section{RESULTADOS Y DISCUSIÓN}

Esta investigación acción buscó desarrollar conocimientos, actitudes y valores, para que los niños y niñas, familia y comunidad del nivel Medio Mayor comprendieran los efectos del cambio climático. A continuación, se analizarán los resultados de acuerdo a las líneas de acción que se trabajaron en la fase de actuar.

\subsection{Niños y niñas:}

Para evidenciar el proyecto de intervención pedagógica 'Conociendo el cambio climático en el Jardín Infantil El Peneca', que la estudiante de práctica profesional implementó, se presentan primero los resultados obtenidos de la observación participante. Con respecto al primer tema, concepto de cambio climático, los niños y niñas señalaron los cambios en el clima como el aumento de las temperaturas y disminución de las lluvias que se producían por el calentamiento global.

En relación al segundo tema, efectos del cambio climático en el Valle de Aconcagua, los párvulos identificaron que las inundaciones y los huracanes eran fenómenos que se producían a consecuencia de las temperaturas extremas. Además, mencionaron que la sobre explotación de vives y paltos estaban provocando la desertificación del suelo y la sequía en el Valle. También los niños y niñas comprendieron que estos efectos eran provocados por las acciones que realizaban las personas.

En cuanto al tema, escasez hídrica, los párvulos fueron comprendiendo la importancia del agua, aprendieron acciones para cuidar el agua: cerrar la llave, lavar las manos con poca agua, usar vaso para cepillarse los dientes y beber agua. Así también reconocieron en la pizarra digital, diversas imágenes dónde se daba cuenta la consecuencia de la sequía. Por otro lado expusieron lo que ocurre con los seres vivos cuando hay escasez de agua.

Sobre el tema, mitigaciones que se realizan en el Valle de Aconcagua, los niños y niñas identificaron las distintas formas de minimizar los residuos como el reciclaje de papel (lámina 1).

\section{Lámina 1: reciclando papel}




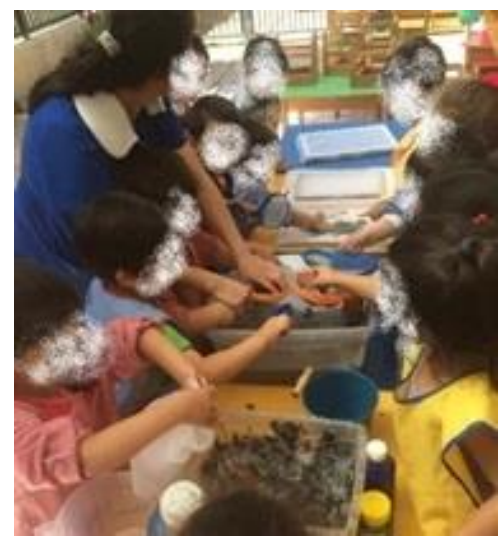

También trabajaron manualidades con distintos residuos como maceteros, flores y palitroques con botellas. Se expresaron creativamente con distintas cajas en la creación de esculturas y trabajaron con diferentes papeles (revistas, pedazos de cartulinas, goma eva y géneros), para decorar folletos informativos.

Además, demostraron la importancia del cultivo orgánico al sembrar condimentos- perejil, cilantro y orégano- que luego se los llevaron a sus hogares (lámina 2).

\section{Lámina 2: sembrando condimentos}

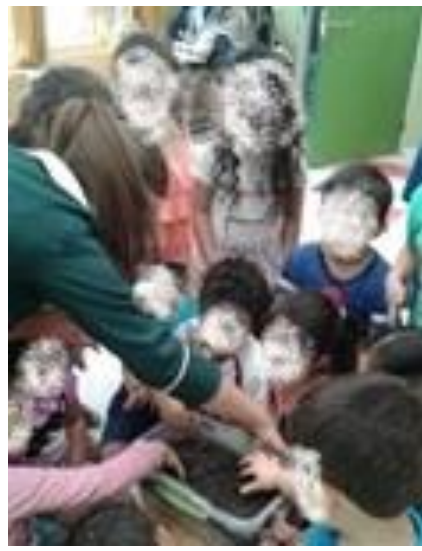

Trabajaron las distintas formas de reutilizar y reducir la ropa haciendo una bolsa para comprar y un plumero. Aprendieron una canción para incentivar el reciclaje de ropa a la familia. Por medio de exposiciones y juegos en la pizarra digital explicaron la importancia de utilizar diferentes artefactos para cuidar la energía como las ampolletas eficientes, apagar las luces y desenchufar los artefactos que no se ocupan.

Los resultados antes señalados evidencian que educar sobre el medio ambiente desde la educación infantil, desarrolla en los niños y niñas conductas y actitudes positivas hacia la naturaleza, estableciendo así un hábito en ellos (Souto-Seijo, Regueiro y Estévez, 2017). En el mismo contexto Cortés y García-Morís dicen que los "niño/as adquieran una conciencia ambiental que les permita actuar razonablemente respecto del planeta" (2014, p. 151), con conciencia y valores para construir un mundo más sostenible (Macedo, 2006).

Continuando con los resultados del trabajo con los niños y niñas, ahora se analizarán la encuesta que se le envío a la familia para que dieran cuenta de los conceptos, valores y actitudes que iban aprendiendo los niños y niñas del cambio climático. Sólo el 54\% de las familias contestaron la encuesta, en el siguiente cuadro se especifican las respuestas de los tópicos evaluados: 
Cuadro 1: Encuesta

\begin{tabular}{|c|c|c|c|}
\hline Familia & $\begin{array}{l}\text { Efectos del cambio climático en el } \\
\text { Valle de Aconcagua }\end{array}$ & Escasez hídrica & $\begin{array}{l}\text { Mitigaciones que se realizan en el } \\
\text { Valle de Aconcagua }\end{array}$ \\
\hline 1 & & Cantaba una canción & $\begin{array}{l}\text { Estamos reduciendo la basura. } \\
\text { Reciclar el papel, cartón y ropa. }\end{array}$ \\
\hline 2 & $\begin{array}{l}\text { Nos explicó porque hace mucho } \\
\text { calor }\end{array}$ & Cuidar el agua & $\begin{array}{l}\text { Reducir, reutilizar y reciclar la } \\
\text { basura. Nos enseñó a reutilizar el } \\
\text { papel. }\end{array}$ \\
\hline 3 & $\begin{array}{l}\text { Comentamos lo que sucede con el } \\
\text { frío en otros lugares }\end{array}$ & $\begin{array}{l}\text { Está más pendiente de cuidar el } \\
\text { agua. }\end{array}$ & $\begin{array}{l}\text { Me ayuda a cuidar las plantas. } \\
\text { Nos enseñó las palabras de } \\
\text { reciclar, reutilizar y reducir. }\end{array}$ \\
\hline 4 & $\begin{array}{l}\text { Nos contó sobre el cambio de } \\
\text { temperaturas. }\end{array}$ & $\begin{array}{l}\text { Está pendiente de cerrar bien las } \\
\text { llaves. }\end{array}$ & $\begin{array}{l}\text { Estamos llevando las botellas de } \\
\text { vidrios al supermercado. Nos } \\
\text { enseñó una canción para reutilizar } \\
\text { y reducir. Estuvimos reciclando } \\
\text { en papel. }\end{array}$ \\
\hline 5 & $\begin{array}{l}\text { Nos habló del calentamiento de la } \\
\text { tierra. }\end{array}$ & Tomar agua en un vaso. & $\begin{array}{l}\text { Cuida de no tirar los papeles en el } \\
\text { parque. Reutilizar las cosas. }\end{array}$ \\
\hline 6 & & Cuidar el agua. & $\begin{array}{l}\text { Estamos reciclando la basura. } \\
\text { Reutilizar, reciclar y reducir. }\end{array}$ \\
\hline 7 & Nos contó sobre la nieve. & & $\begin{array}{l}\text { Estamos separando la basura y } \\
\text { llevándola al supermercado. Nos } \\
\text { habló de reutilizar las cosas. }\end{array}$ \\
\hline 8 & $\begin{array}{l}\text { Habla sobre los cambios de } \\
\text { temperatura. }\end{array}$ & Debemos cuidar el agua. & $\begin{array}{l}\text { Riega las plantas y está pendiente } \\
\text { en poner las botellas en la canasta. } \\
\text { Reciclar y ordenar la basura. }\end{array}$ \\
\hline 9 & Converso sobre el calor. & $\begin{array}{l}\text { No debemos dejar las llaves } \\
\text { abiertas. }\end{array}$ & $\begin{array}{l}\text { Cuida las plantas y su perro. } \\
\text { Reducir, reutilizar y reciclar. }\end{array}$ \\
\hline 10 & $\begin{array}{l}\text { Tenet cuidado con el sol en la } \\
\text { playa. }\end{array}$ & Cuidar el agua. & $\begin{array}{l}\text { Cuidar las plantas. Reducir, } \\
\text { reutilizar y reciclar. }\end{array}$ \\
\hline 11 & & $\begin{array}{l}\text { Está pendiente en cuidar el } \\
\text { agua. }\end{array}$ & $\begin{array}{l}\text { Botar la basura dónde } \\
\text { corresponde y llevar lo que se } \\
\text { puede al supermercado para } \\
\text { reciclar. Reducir, reutilizar y } \\
\text { reciclar. }\end{array}$ \\
\hline 12 & $\begin{array}{l}\text { Cuidar el planeta por el } \\
\text { calentamiento. }\end{array}$ & & $\begin{array}{l}\text { Botar la basura en el basurero y } \\
\text { llevar los plásticos al contenedor } \\
\text { que está cerca del jardín. Reducir, } \\
\text { reutilizar y reciclar. }\end{array}$ \\
\hline 13 & Cuidarnos del sol. & Cuidar el agua. & $\begin{array}{l}\text { Cuida las plantas y los animales. } \\
\text { Reducir, reutilizar y reciclar. }\end{array}$ \\
\hline 14 & & $\begin{array}{l}\text { Dar agua a los animales en } \\
\text { pocillos. }\end{array}$ & $\begin{array}{l}\text { Pintamos tarros y estamos } \\
\text { reciclando la basura. } \\
\text { Nos enseñó cuidar los animales y } \\
\text { las plantas. Reducir, reutilizar y } \\
\text { reciclar. }\end{array}$ \\
\hline 15 & Nos habló sobre las temperaturas. & Cuidar el agua. & $\begin{array}{l}\text { Cuidar el medio ambiente } \\
\text { reciclando las botellas. Estamos } \\
\text { tirando la basura en puntos de } \\
\text { reciclaje. } \\
\text { Nos explicó como reciclar el } \\
\text { papel. }\end{array}$ \\
\hline
\end{tabular}

Las respuestas dadas por las familias, evidencian que los niños y niñas iban informando los distintos aprendizajes que fueron construyendo en el proyecto. La mayoría de los párvulos les señalaron a sus padres que el cambio climático provoca alteraciones en las temperaturas, que había que cuidar el agua, donde y como reciclar los desechos, reutilizar y cuidar el medio ambiente. Según Schindel (2012), los programas de acción ambiental empoderan a las comunidades para que sean más consientes con la naturaleza. Además los proyectos educativos favorecen la curiosidad y 
el cuestionamiento de los fenómenos que observan, potenciando las habilidades científicas (Pérez y Caldeiro, 2016).

\subsection{Familia:}

El trabajo que se desarrolló con la familia fue informativo, en las dos reuniones que intervino la estudiante en práctica pudo observar gran interés de los padres, madres y abuelas, por conocer las distintas formas de manejar los residuos en sus hogares. Así también la participación activa que tuvieron los familiares de los párvulos para trabajar el uso eficiente del agua. Estos contenidos fueron reforzados con los tres trípticos que se enviaron a sus hogares. Para el Ministerio del Medio Ambiente se deben hacer distintas acciones de mitigación para afrontar a nivel local el cambio climático. Asimismo, la UNESCO señala que se deben desarrollar "competencias, actitudes y comportamientos para un planeta más sostenible e inclusivo" (2017, p.1).

\subsection{Comunidad:}

El proyecto implementado consideró el trabajo con la comunidad, los niños y niñas elaboraron con materiales reciclados diferentes folletos informativos, los que fueron entregados por ellos. A medida que visitaban las viviendas, los párvulos realizaron conversaciones con los vecinos de los contenidos aprendidos en las experiencias pedagógicas, esta instancia le permitió observar a la estudiante de práctica los contenidos aprendidos y el interés de los vecinos por los temas tratados, para Asencio (2017), trabajar en equipo la alfabetización científica mejorará el desarrollo sostenible.

\section{CONCLUSIÓN}

El objetivo de esta investigación-acción fue desarrollar conocimientos, actitudes y valores, para comprender los efectos del cambio climático en los niños y niñas del nivel Medio Mayor. Por medio del proyecto 'Conociendo el cambio climático en el Jardín Infantil El Peneca' la estudiante de práctica profesional realizó experiencia de aprendizajes a los niños y niñas, familia y comunidad.

Las EP implementadas a los niños y niñas les permitió conocer el concepto de cambio climático y los efectos que han provocado en el Valle de Aconcagua. A su vez se desarrollaron actitudes para que los párvulos valoren el agua y el uso responsable y solidario de este recurso. También se trabajó medidas de mitigación: minimizar residuos de papel, botellas plásticas, cajas de cartón, goma eva y géneros; reutilizar y reducir ropa; ahorra energía; y hacer cultivos orgánicos.

El trabajo con la familia los educó en el manejo de residuos, la importancia del agua y su uso responsable y las acciones que pueden hacer en sus hogares para ahorra energía, se realizó en dos reuniones en el establecimiento educativo y se enviaron tres trípticos educativos. También se realizó una visita educativa a la comunidad cercana al Jardín Infantil, los niños y niñas entregaron folletos y dialogaron con los vecinos de los temas trabajados en el proyecto.

Los precursores de la Educación Parvularia como Decroly, Fröbel, Pestalozzi y Montessori consideraron al medio natural como ambiente fundamental para el aprendizaje, ya que la interacción de los niños y niñas con el medio ambiente les permite su desarrollo integral. Por consiguiente, educar para mantener el medio ambiente es fundamental desde de la Educación Parvularia, que los niños y niñas "contribuyan en sus acciones al cuidado del ambiente natural y su biodiversidad, en una perspectiva de la sostenibilidad” (Subsecretaria de Educación Parvularia, 2017, p. 82). 
La limitación que tuvo este estudio fue el tiempo de implementación del proyecto, esto se debe a que la práctica profesional dura sólo un semestre. Habría sido interesante realizar una investigación con un tiempo más largos para analizar otros aspectos. En relación a lo anterior es que se propone como proyecciones de esta investigación, implementar un proyecto que dure entre un a dos años, para responder otras preguntas que nacen de los resultados obtenidos. Por ejemplo ¿qué cambios efectivos realizaron las familias para mitigar el cambio climático? ¿Cuál es la percepción que tienen los niños y niñas, la familia y la comunidad sobre el cambio climático? ¿Cuáles son las limitaciones que tiene la familia para hacer cambios en el cuidado del medio ambiente?

\section{REFERENCIAS}

Arias, F. (2016). El Proyecto de Investigación: Introducción a la Metodología Científica (6a. ed.). Caracas, Venezuela: Episteme, Editorial C.A.

Asencio, E. (2013). Acercamiento a la formación de docentes de ciencias en Latinoamérica. Experiencias en el contexto cubano. Revista Eureka sobre Enseñanza y Divulgación de las Ciencias, 10(3).

Asencio, E. (2017). La educación científica: percepciones y retos actuales. Educación y Educadores, 20(2), $282-296$. DOI: 10.5294/edu.2017.20.2.7

Cortés, C, y García-Morís, R. (2014). Propuesta metodológica para una didáctica del medio ambiente en educación infantil: la investigación sobre cuentos ambientales. Capítulo de libro Nuevas perspectivas conceptuales y metodológicas para la educación geográfica. Editado Grupo de Didáctica de la Geografía de la Asociación de Geógrafos Españoles.

Creswell, J. (2012). Educational research. Planning, conducting and evaluating quantitative and qualitative research. [Investigación educativa. Planeación, conducción y evaluación en investigación cuantitativa y cualitativa]. ( $4^{\mathrm{a}}$ ed). USA: Pearson.

Estenssoro, F. (2010). Crisis ambiental y cambio climático en la política global: un tema crecientemente complejo para América Latina. Universum, 25(2), 57-77. doi.org/10.4067/S0718-23762010000200005.

González, F., Arias, H. y Ávila, E. (2021). Aprender sobre el medio ambiente: una propuesta de micros audiovisuales para la etapa preescolar. Revista Ibero-americana de Educação, 85 (1), 135-157.

Hernández, R. Fernández, C. y Baptista, P. (2014). Metodología de la investigación (6a ed.). México: McGraw-Hill. Recupeado de http://upla.edu.pe/portal/wp-content/uploads/2017/01/Hern\%C3\%A1ndez-R.-2014Metodologia-de-la-Investigacion.pdf.pdf

Junta Nacional de Jardines Infantiles (2015). Lineamientos técnicos: marco técnico pedagógico para la mejora de la calidad de las prácticas educativas. Departamento Técnico-Pedagógico, Santiago, Chile.

Junta Nacional de Jardines Infantiles. Recuperado De: https://www.junji.gob.cl/quienes-somos /mision/ [Fecha de consulta: octubre, 2019].

Macedo, B. (2006). Habilidades para la vida: contribución desde la educación científica en el marco de la década de la educación para el desarrollo sostenible. Revista Educación, 119, 2-7.

Ministerio de Educación (2013). Desarrollo Sustentable en Educación Parvularia experiencias de aprendizaje en NT1 y NT2. Santiago, Chile: MINEDUC.

Ministerio del Medio Ambiente (2018). Tercer informe bienal de actualización de Chile sobre cambio climático 2018. Santiago, Chile.

Organización de Estados Iberoamericanos para la Educación, la Ciencia y la Cultura (OEI). (2010). 2021 Metas Educativas. La Educación que queremos para la generación de los Bicentenarios. Madrid, España.

Organización de las Naciones Unidas. (1987). Informe de la Comisión Mundial sobre el Medio Ambiente y el Desarrollo «Nuestro futuro común».

Organización de las Naciones Unidas. (1992). Protocolo de Kyoto. Convención Marco de las Naciones Unidas sobre Cambio Climático. Nueva York, USA. 
Organización de las Naciones Unidas. (2015). Transformar nuestro mundo: la Agenda 2030 para el Desarrollo Sostenible.

Organización de las Naciones Unidas. Disponible en: https://unstats.un.org/sdgs/report/2019/The-SustainableDevelopment-Goals-Report-2019_Spanish.pdf

Organización de las Naciones Unidas para la Educación, la Ciencia y la Cultura. (2017). Informe de seguimiento de la educación en el mundo. 2016. La educación al servicio de los pueblos y el planeta: Creación de futuros sostenibles para todos. París, Francia: Autor

Pérez, S. y Caldeiro, M. (2016). Aprendiendo ciencia en el Aula Didáctica: retos y potencialidades. Revista Ecos de la Academia, 3, 182- 185.

Pérez-Lisboa, S. (2017). Descubriendo el lenguaje a través de la realidad aumentada y la pizarra digital. Revista Electrónica Educare (Educare Electronic Journal), 21(3), 1-13. doi: http://dx.doi.org/10.15359/ree.21-3.14

Préndez, M. \& Calderón, V. (2013). Análisis de Contaminantes en la Cuenca del Río Aconcagua en Chile. Evaluación de Riesgo Humano y Ambiental Información Tecnológica, 24(1).

Sabatini, F. (1994). Espiral histórica de conflictos ambientales: el caso de chile. Ambiente y desarrollo, X(4), 15-22.

Sandín, M. P. (2003). Investigación cualitativa en educación: Fundamentos y tradiciones. Madrid, España: McGraw-Hill Interamericana de España.

Schindel, A. (2012). Student empowerment in an environmental science classroom: Toward a framework for social justice science education. Science Education, 96(6), 990-1012. Doi: 10.1002/sce.21035.

Souto-Seijo, A., Regueiro, B, y Estévez, I. (2017). Propuesta didáctica de Educación Ambiental en Educación Infantil. Revista de Estudios E Investigación en Psicología y Educación, Extr. (5). DOI: https://doi.org/10.17979/reipe.2017.0.05.2134

Stringer, E. T. (1999). Action research: A handbook for practitioners (2a Ed.). Thousand Oaks, CA, EE. UU.: Sage. Subsecretaria Educación Parvularia (2017). Bases Curriculares de Educación Parvularia. Santiago, Chile.

Toro, B. Ugarte, A. (2018). Educación para el desarrollo sustentable. Ediciones JUNJI, Santiago, Chile. 